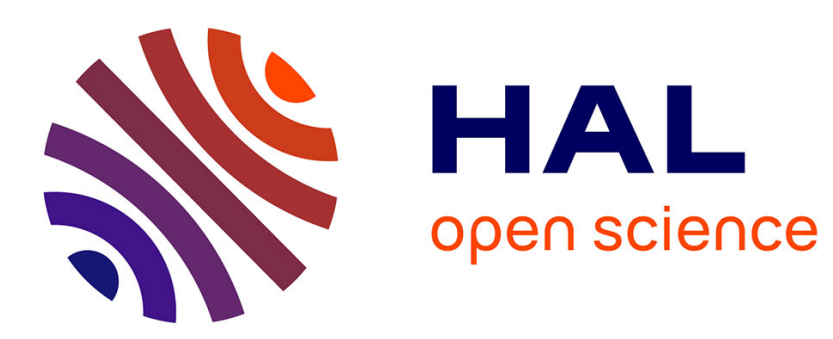

\title{
Simulations of Modulation of the Current in a Field Emitter Caused by a CW or Pulsed Laser
}

M. Hagmann

\section{To cite this version:}

M. Hagmann. Simulations of Modulation of the Current in a Field Emitter Caused by a CW or Pulsed Laser. Journal de Physique IV Proceedings, 1996, 06 (C5), pp.C5-71-C5-76. 10.1051/jp4:1996511. jpa-00254390

\section{HAL Id: jpa-00254390 https://hal.science/jpa-00254390}

Submitted on 1 Jan 1996

HAL is a multi-disciplinary open access archive for the deposit and dissemination of scientific research documents, whether they are published or not. The documents may come from teaching and research institutions in France or abroad, or from public or private research centers.
L'archive ouverte pluridisciplinaire HAL, est destinée au dépôt et à la diffusion de documents scientifiques de niveau recherche, publiés ou non, émanant des établissements d'enseignement et de recherche français ou étrangers, des laboratoires publics ou privés. 


\title{
Simulations of Modulation of the Current in a Field Emitter Caused by a CW or Pulsed Laser
}

\author{
M.J. Hagmann \\ Department of Electrical and Computer Engineering, Florida International University, Miami, FL 33199, \\ U.S.A.
}

\begin{abstract}
Solutions of the time-dependent Schrödinger equation show that the tunneling current in a field emitter may be modulated by means of a laser. The steady-state response is calculated with Floquet methods, and the transient response is determined with a product formulation that does not require the use of absorbing boundary conditions or wave packets. The steady-state response contains a series of resonances, but when the full distribution of energies in a metal is considered the resulting effect is a broad peak that is typically centered in the blue end of the visible spectrum, but may be shifted by varying the applied static field. The transient solutions show that the response of the electrons to the optical fields is delayed by the semiclassical tunneling time, which is defined as the time for traversing the inverted barrier.
\end{abstract}

\section{INTRODUCTION}

Simulations of quantum tunneling with time-dependent barriers show a series of resonances at which there is a marked increase in the tunneling current [1]. For the special case of square barriers these resonances occur when tunneling particles absorbing quanta from the time-dependent source are above the barrier and the barrier length is an integral multiple of one-half the (then real) de Broglie wavelength. The mechanism for resonance, which is the same as that for the transmission of particles which initially have energies exceeding the height of the barrier, is reinforcement of the wave function by reflections at the ends of the barrier. The mechanism for resonance appears to be the same with barriers having non-square profiles, but it is simpler to determine the wavelengths for resonance with square barriers because the potential is a constant and the turning points are well defined. The present study was made in order to determine how these resonances may relate to laserassisted field emission.

\section{METHODS OF ANALYSIS}

We begin the analysis with the dipole approximation [2] of the time-dependent Schrödinger equation for an electron in a static potential plus a radiation field,

$$
i \hbar \frac{\partial \Psi}{\partial t}=-\frac{\hbar^{2}}{2 m} \nabla^{2} \Psi+\left[V_{0}(\boldsymbol{r})-e \boldsymbol{r} \cdot \boldsymbol{E}(\boldsymbol{r}, t)\right] \Psi
$$

where $V_{o}(\mathbf{r})$ is the static potential and $\mathbf{E}(\mathbf{r}, t)$ is the electric field intensity of the radiation field.

In determining the steady-state response to a laser we set $\mathbf{E}(\mathrm{x}, \mathrm{t})=\mathrm{E}_{1} \cos (\omega \mathrm{t}) \boldsymbol{x}$, where $\mathrm{E}_{1}$ is a constant. Density functional theory [3] is used to determine the self-consistent static potential $V_{0}(\mathbf{r})$ for a given applied static field and a set of energies representing the distribution in a one-dimensional jellium model of the metal. Floquet's theorem may be used [4] to show that for each energy $\mathrm{E}$ the solution of Eq. (1) may be written as 


$$
\Psi=\sum_{-\infty}^{\infty} F_{n}(x) \exp [-i(E+n \hbar \omega) t / \hbar]
$$

where the functions $F_{n}(x)$ must be determined. The time-dependence in Eq. (2) shows that tunneling electrons exchange quanta with the laser [5-6]. We substitute Eq. (2) into Eq. (1) and require that the resulting expression be satisfied as an identify for all values of time, thus obtaining the following ordinary differential equation:

$$
\frac{-\hbar^{2}}{2 m} F_{n}^{\prime \prime}+\left(V_{0}-E-n \hbar \omega\right) E_{n}+\frac{1}{2}\left(E_{n-1}+E_{n+1}\right) E_{1} x=
$$

We solve Eq. (3) to determine the functions $F_{n}(x)$ by means of shooting methods [7] in order to avoid forming a large matrix, and then superimpose the probability densities at each simulated energy for a weighted sum. For the case of no laser Eq. (3) reduces to the time-independent Schrödinger equation.

In determining the response to a laser pulse, we set $E(x, t)=E_{1} \sin (\omega t) U(t) x$, where $E_{1}$ is a constant and $U(t)$ is the unit step function ( 1 for $t>0$, else 0 ). We find it convenient to use a product formulation in which the function $F(r, t)$ is defined such that $\Psi(\mathbf{r}, t)=F(r, t) \Phi(r) \exp (-i E t / \hbar)$, where $\Phi(r) \exp (-i E t / \hbar)$ is the solution without the radiation field. Substituting into Eq. (1) results in the following equation:

$$
\frac{\partial F}{\partial t}=\frac{i \hbar}{2 m} \nabla^{2} F+\frac{i \hbar}{m \Phi} \nabla \Phi \cdot \nabla F+\frac{i e r \cdot E(r, t) F}{\hbar}
$$

For the case of no laser $F=$ constant is a solution of Eq. (4), and the boundary condition $(F(r, t)=1$ for $t<0$ ) requires that the constant is unity. Density functional theory is used to determine the self-consistent static potential $V_{0}(\mathbf{r})$ and $\Phi(\mathbf{r})$ for a given applied static field and a set of energies representing the distribution in a one-dimensional jellium model of the metal. The following finite difference approximation of Eq. (4) is obtained by using the midpoint method [8] to approximate the time derivative of $F(x, t)$ :

$$
\begin{aligned}
F_{I, J+1} & =F_{I, J-1} \\
& +\frac{i \hbar \Delta t}{m(\Delta x)^{2}}\left[F_{I+1, J}-2 F_{I, J}+F_{I-1, J}\right] \\
& +\frac{i \hbar \Delta t}{m \Delta x}\left[\frac{1}{\Phi} \frac{d \Phi}{d x}\right]_{I}\left[F_{I+1, J}-E_{I-1, J}\right] \\
& +\frac{2 i \Delta t I \Delta x e E_{1}}{\hbar} \sin (\omega J \Delta t) U(J) F_{I, J}
\end{aligned}
$$

where $F_{L,}$ is defined by $F(I \cdot \Delta x, J \cdot \Delta t)$.

The transient solutions for the response to a laser pulse are obtained in the following manner: (1) First density functional theory is used to determine $V_{0}(x)$ and $\Phi(x)$ for a set of energies representing the distribution in the metal. (2) Next Eq. (5) is solved by time stepping from $t=0$ where $F_{L, 0}$ is identically unity. (3) Then the wave function $\Psi(x, t)$ is calculated for each energy $E$ by using the definition $\Psi(x, t)=$ $\mathrm{F}(\mathrm{x}, \mathrm{t}) \Phi(\mathrm{x}) \exp (-\mathrm{iEt} / \mathrm{h})$. (4) Finally, the probability density is determined by adding the values for the different energies in the distribution. This implementation does not require the use of absorbing boundary conditions or wave packets [9] and is explicit, so it does not require large matrices that are necessary with split-operator formulations [10]. When the radiation field is not too strong, when compared with the static potential, it is possible to use values of $\Delta \mathrm{x}$ with the product formulation that are much larger than when solving for the wave function directly, because then $\mathrm{F}(\mathrm{x}, \mathrm{t})$ varies more slowly in space than does $\Psi(\mathrm{x}, \mathrm{t})$. While the present method is not unconditionally stable, the solutions have been shown to be stable over the range of simulated durations in the examples. The steady-state and transient solutions described in this paper are not completely selfconsistent because the static potential is not corrected to allow for changes in the charge distribution caused by the radiation field. However, a partial correction for this effect is made by adjusting the incident electric 
field to allow for screening by the metal [11]. We have found that it is necessary to discretize to a depth considerably greater than the plasma wavelength, for example $100 \mathrm{~nm}$ in tungsten metal for which $\lambda_{\mathrm{p}} \approx 54.2$ nm.

\section{SIMULATIONS OF MODULATION CAUSED BY A CW LASER}

Figure 1 shows the ratio of the tunneling current with a laser to that with no laser as a function of the optical wavelength for a tungsten surface with an applied static field of $5 \mathrm{~V} / \mathrm{nm}$ and a laser power flux density of $10^{10} \mathrm{~W} / \mathrm{m}^{2}$. The current ratios are shown separately for electrons having normal energies from 0.95 to 1.00 (from top to base of the figure) of the Fermi level in tungsten. We have previously noted that for the case of square barriers, resonances occur when tunneling particles absorbing quanta are above the barrier and the barrier length is an integral multiple of one-half the de Broglie wavelength. As noted in the introduction, it is not as simple to calculate the wavelengths for resonances with barriers having nonsquare profiles. However, the energy dependence of the wavelength for resonance and the current ratio at resonance, as seen in Fig. 1, are consistent with the observation that electrons with less energy require more energetic photons in order to be promoted above the barrier. The increase in transmission caused by the radiation field is proportional to the power flux density, which is consistent with the mechanism being the absorption of quanta. All other simulations of modulation caused by a $\mathrm{CW}$ laser were made using the full distribution of energies in the metal so the resonances seen in the following figures are less pronounced than those seen in Fig. 1 because of this superposition. Typically 100 energies were used to simulate this distribution.

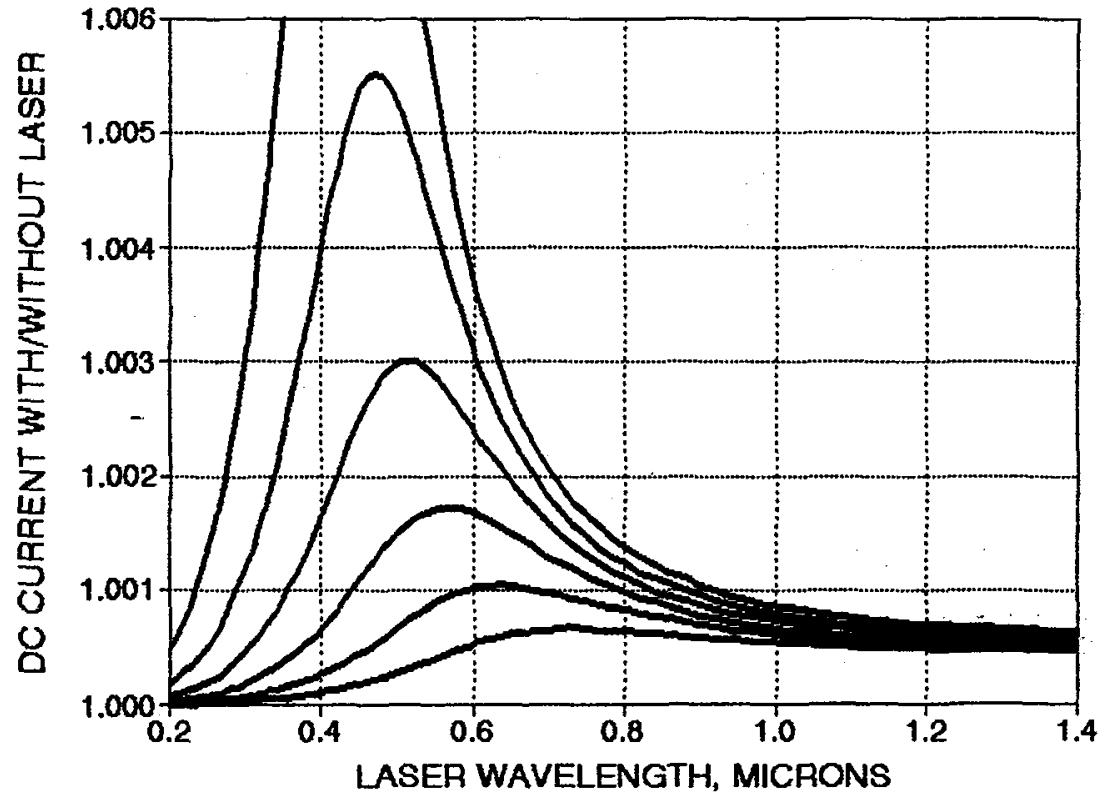

Figure 1: Ratio of current with/without a laser as a function of optical wavelength for electrons with normal energies of $0.95,0.96$, $0.97,0.98,0.99$ and $1.00 \mathrm{E}_{\mathrm{f}}$ (top to base) in tungsten with an applied static field of $5 \mathrm{~V} / \mathrm{nm}$ and a power flux density of $10^{10} \mathrm{~W} / \mathrm{m}^{2}$.

Figure 2 shows the ratio of the tunneling current with a laser to that with no laser as a function of the optical wavelength for a tungsten surface with an applied static field of $5 \mathrm{~V} / \mathrm{nm}$ and a laser power flux density of $10^{10} \mathrm{~W} / \mathrm{m}^{2}$. The full distribution of energies in metal was simulated, and the current ratios are shown separately for tungsten at temperatures from -273 to $3000^{\circ} \mathrm{C}$ (from top to base of the figure). It may be seen in the figure that the resonance is more prominent at lower temperatures, and this may be understood because of the more narrow distribution of energies at the lower temperatures. 


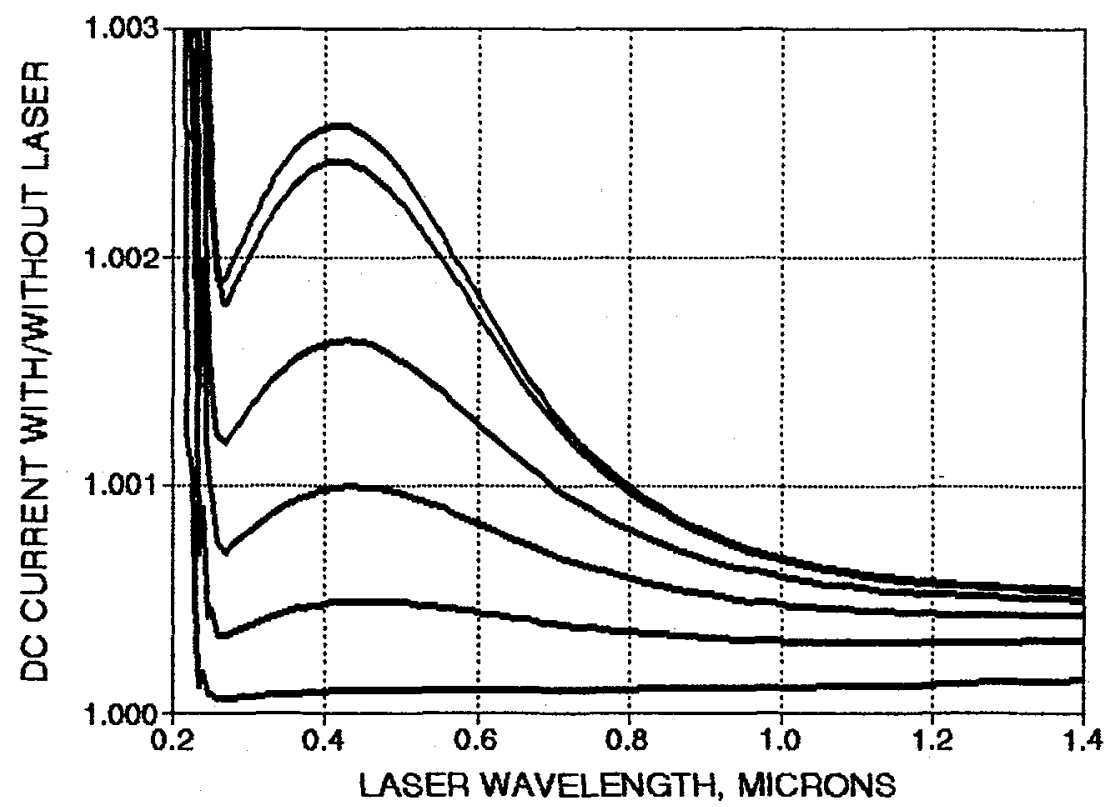

Figure 2: Ratio of current with/without a laser as a function of optical wavelength for a tungsten surface at $-273,200,1000,1500$, 2000 and $3000^{\circ} \mathrm{C}$ (top to base) with an applied static field of $5 \mathrm{~V} / \mathrm{nm}$ and a power flux density of $10^{10} \mathrm{~W} / \mathrm{m}^{2}$.

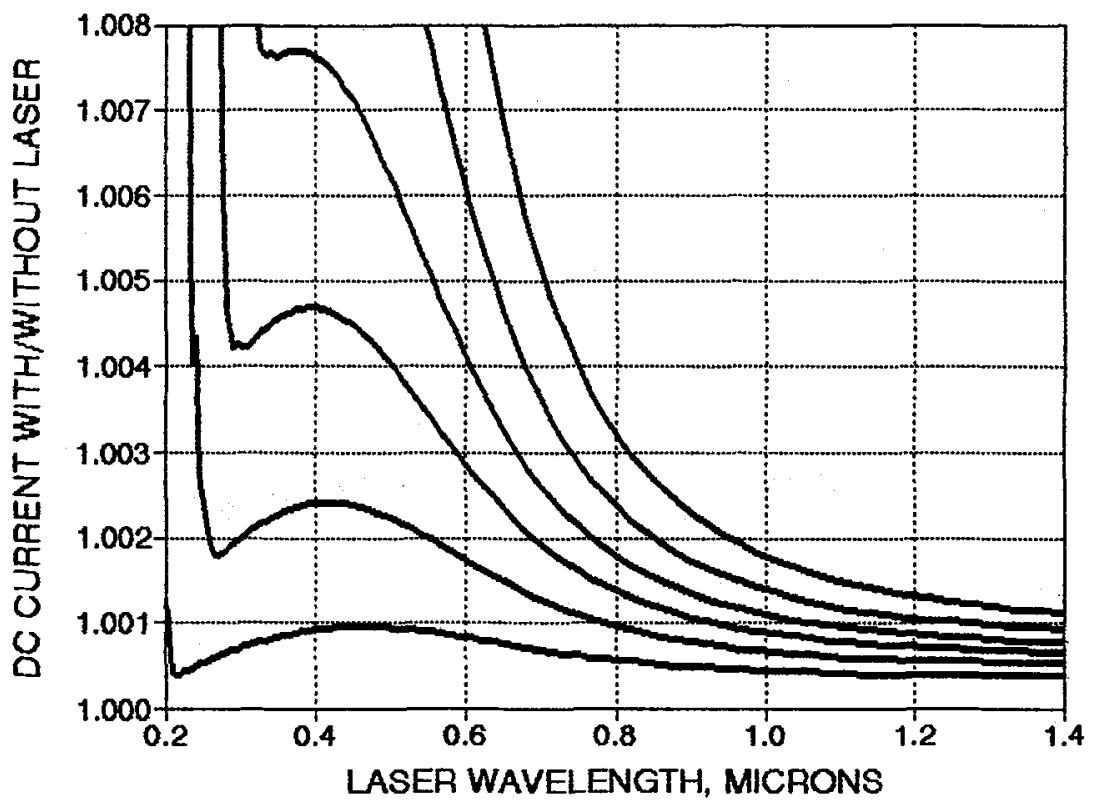

Figure 3: Ratio of current with/without a laser as a function of optical wavelength for a tungsten surface at $200^{\circ} \mathrm{C}$ with applied static fields of $4.1,4.3,4.5,4.7,5.0$ and $5.5 \mathrm{~V} / \mathrm{nm}$ (top to base) and a power fiux density of $10^{10} \mathrm{~W} / \mathrm{m}^{2}$. 
Figure 3 shows the ratio of the tunneling current with a laser to that with no laser as a function of the optical wavelength for a tungsten surface at $200^{\circ} \mathrm{C}$ with a laser power flux density of $10^{10} \mathrm{~W} / \mathrm{m}^{2}$. The current ratios are shown separately for applied static fields from 4.1 to $5.5 \mathrm{~V} / \mathrm{nm}$ (from top to base of the figure). The effect of the applied static field on the wavelength for resonance, and the current ratio at resonance, are consistent with the observation that raising the barrier height requires more energetic photons in order for the electrons to be promoted above the barrier.

\section{SIMULATION OF THE INITIAL RESPONSE TO A LASER PULSE}

Figure 4 shows the transient response of electrons to a laser $(\lambda=0.58 \mu \mathrm{m})$ that illumines a tungsten surface having an applied static field of $5 \mathrm{~V} / \mathrm{nm}$ with a power flux density of $10^{10} \mathrm{~W} / \mathrm{m}^{2}$ beginning at time $t=0$. The value of $\mathrm{FF}^{*}$, the ratio of the probability density to the static value, is plotted as a function of position and time. The static potential $V_{0}(x)$ used in this simulation was obtained using density functional theory with a one-dimensional jellium model of a tungsten surface subjected to the applied static field. However, computational restraints did not permit updating the potential to correct for the changes in charge distribution caused by the radiation field, and limited the calculations to one value of normal energy, which was set equal to 0.98 of the Fermi level in tungsten. Only the values in vacuum are shown, and the left rear edge of Fig. 4 corresponds to the tungsten/vacuum interface. The phasing of the optical electric field was chosen such that a node in the sinusoid occurred at time $t=0$.

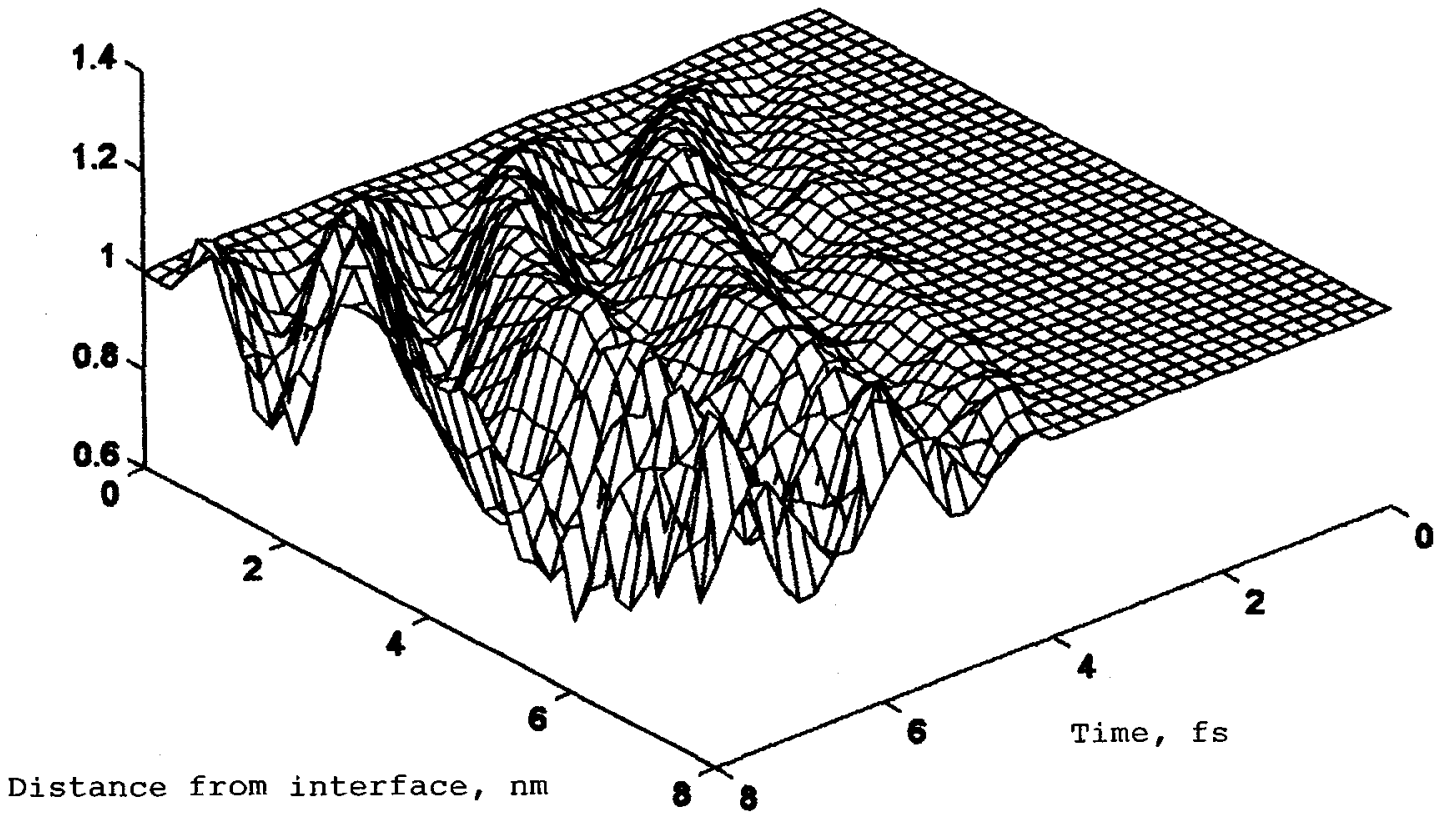

Figure 4: Ratio of probability density to the static value for $0.98 \mathrm{E}_{\mathrm{f}}$ electrons in vacuum near a tungsten surface having an applied static field of $5.0 \mathrm{~V} / \mathrm{nm}$ and a radiation field with $\lambda=0.58 \mu \mathrm{m}$ and a power flux density of $10^{10} \mathrm{~W} / \mathrm{m}^{2}$ beginning at time $t=0$.

The period of the optical signal $\tau=1.935 \mathrm{fs}$, and the semiclassical value of the tunneling time, defined as the time for traversing the inverted barrier [12] is $T_{s c}=1.435 \mathrm{fs}$ in this example. Careful examination of the data in Fig. 4 shows that there is no response to the radiation field until the time $T_{s c}$ is exceeded. The barrier height is sequentially increased and decreased due to the superposition of the electric vector of the radiation field with the static potential. Following the initial delay of $\mathrm{T}_{\mathrm{sc}}$, each decrease in the barrier height causes an increase in the current, and each increase in the barrier height causes a decrease in the current. However, there is a delay of $T_{\mathrm{sc}} / 2$ between each extremum in the barrier height and the corresponding extremum in the 
probability density as the tunneling process is completed. Consecutive peaks in the current increase in magnitude during the $8 \mathrm{fs}$ of the simulation to approach the steady-state solution at the resonance. Acceleration of the electrons due to the applied static field is also evident in Fig. 4.

\section{DISCUSSION AND CONCLUSIONS}

The steady-state solutions in this paper are consistent with experimental studies of photofield emission [13] in that the increase in current caused by a laser is 1) proportional to the power flux density, 2) maximum for polarization normal to the tip surface, 3) for electrons that absorb one photon, and 4) more significant near the blue than the red end of the visible spectrum. However, Lee and Robins [14] measured the rise time for the current under the conditions used in photofield emission, and their measurements suggest that thermal effects are present in the experimental data. Their simulations show that the thermal relaxation time is strongly dependent on the shank length and the size of the focal spot, and thus may vary from a few $\mu$ s to a few ms. Mechanical choppers have been used to decrease the effects of heating, and it is claimed [15] that the thermal component of the photocurrent is reduced by 40 percent by chopping at a frequency of $8.5 \mathrm{kHz}$. The present analysis suggests that radiofrequency modulation would be a useful tool for separating thermal from non-thermal effects when studying photofield emission.

The delays present in the transient solution are consistent with observations regarding limits to the adiabaticity of the field emission process [16], so that this analysis offers promise as a new method to approach the yet unsolved problem [17] of evaluating the duration of quantum tunneling.

\section{Acknowledgments}

We acknowledge the assistance of J. G. Krasnow in editing, helpful discussions with H. F. Gray and J. L. Shaw (NRL), and support with graphics software by $\mathrm{H}$. Ghassemi (FIU). The Pentium PC used for the calculations was made possible by NSF research equipment grant No. 9500007.

\section{References}

[1] Hagmann, M. J., J. Appl. Phys. 78 (1995) 25-29.

[2] Faisal, F. H. M., Theory of Multiphoton Processes (Plenum, New York, 1987), pp. 8-10.

[3] Lang, N. D. in Solid State Physics, 28, H. Ehrenreich, F. Seitz and D. Turnbull Eds. (Academic Press, New York, 1973), pp. 225-300.

[4] Hagmann M. J., J. Vac. Sci. Technol. B 13 (1995) 1348-1352.

[5] Büttiker, M. and Landauer, R., Physica Scr. 32 (1985) 429-434.

[6] Tien, P. K. and Gordon, J. P., Phys. Rev. 129 (1963) 647-651.

[7] Keller, H. B., Numerical Methods for Two-Point Boundary-Value Problems (Dover, New York, 1992) pp. 39-71.

[8] Young, D. M. and Gregory, R. T., A Survey of Numerical Mathematics, Vol. I (Addison-Wesley, Reading MA, 1972) pp. 453 456.

[9] Macias, D., Brouard, S., and Muga, J. G., Chem. Phys. Lett. 228 (1994) 672-674.

[10] Kulander, K. C., Phys Rev A 35 (1987) 445-447.

[11] Ng, A., Celliers, P., Forsman, A., More, R. M., Lee, Y. T., Perrot, F., Dharma-wardana, M. W. C., and Rinker, G. A., Phys. Rev. Lett. 72 (1994) 3351-3354.

[12] Hagmann, M. J., Int. J. Quantum Chem. 52, Quantum Symp. Issue No. 28 (1994) 271-281.

[13] Gao, Y., and Reifenberger, R., Phys. Rev. B 35 (1987) 6627-6636.

[14] Lee, M. J. G. and Robins, E. S., J. Appl. Phys. 65 (1989) 1699-1706.

[15] Radoń, T. and Jaskólka, S., Surf. Sci. 231 (1990) 160-164.

[16] Fursey, G. N., J. Vac. Sci. Technol. B 13 (1995) 558-565.

[17] Landauer, R. and Martin, Th., Rev. Mod. Phys. 66 (1994) 217-228. 